\title{
Heterogeneidade na estrutura e diversidade de árvores de cabrucas no centro-sul do Estado da Bahia, Brasil
}

\author{
Rui Barbosa Alves da Silva Guimarães ${ }^{1,2,5}$, Paulo Sávio Damásio da Silva ${ }^{3}$ e \\ Michele Martins Corrêa ${ }^{4}$
}

Recebido: 30.05.2016; aceito: 1.03.2017

\begin{abstract}
Tree heterogeneity and diversity in cabrucas in central South of Bahia State, Brazil). The contribution of managed ecosystems for biodiversity conservation has been highlighted by recent studies. Tree species diversity was investigated in Cabruca (a traditional agroforest with cocoa and native trees) of ten cocoa farms in central- Southern Bahia state to quantify the remnant tree diversity and to verify if it is influenced by the size of the farm and the size of the Cabruca. A total of 273 trees, 55 trees species and 20 plant families were recorded. From these, $89 \%$ of plant species and $73 \%$ of trees were native species. In average, 23 to 60 tree species and 40 to 236 individuals per hectare were registered. The Shannon-Wiener (H') index varied from 1.3 to 2.5. The mixture coefficient of Jentsch (QM) obtained was two individuals per species. A high heterogeneity was observed among the Cabrucas studied. Tree diversity was not correlated, however, with the total area of the farms or the area of Cabruca on the farms. Our results indicate that Cabruca are variable in tree density and diversity. Additionally, management performed by cocoa farm owners is the main factor that influences the tree community attributes in this agroforestry.
\end{abstract}

Keywords: Agroforestry systems, Atlantic forest, cocoa tree, managed ecosystem, native species

RESUMO - (Heterogeneidade na estrutura e diversidade de árvores de cabrucas no centro-sul do Estado da Bahia, Brasil). O objetivo deste trabalho foi conhecer a diversidade arbórea de cabrucas de fazendas produtoras de cacau no centro-sul da Bahia e verificar se fatores como as áreas das propriedades e das cabrucas influenciam as comunidades arbóreas remanescentes. Foram encontrados 273 indivíduos, pertencentes a 55 espécies e 20 famílias, sendo que $89 \%$ das espécies e $73 \%$ dos indivíduos são nativos da região. Em média, por hectare, foram registrados 23 a 60 espécies arbóreas e 40 a 236 indivíduos. O índice de Shannon-Wiener (H') variou de 1,3 a 2,5 e o quociente de mistura de Jenstch resultou em uma proporção de dois indivíduos para cada espécie. As cabrucas estudadas apresentaram alta heterogeneidade. Contudo, a diversidade arbórea não foi correlacionada com a área total e nem com a área de cabruca nas propriedades. Nossos resultados indicam que as cabrucas são muito variáveis em densidade e diversidade e que o sistema de manejo realizado pelos proprietários das fazendas parece ser o fator que mais influencia os atributos da comunidade arbórea nestas agroflorestas.

Palavras-chave: Agrossilvicultura, cacau, ecossistemas manejados, espécies nativas, Mata Atlântica

\section{Introdução}

A conversão de florestas tropicais em outros tipos de habitat como pequenos fragmentos, pastagens ou áreas agrícolas, com o objetivo de acessar bens de consumo providos pela biodiversidade ou para utilização do espaço, têm alcançado elevadas taxas nos últimos anos e causado a extinção de inúmeras espécies (Harvey et al. 2008, Santos et al. 2008, Cassano et al. 2009, Piasentin \& Gois 2016, Rolim et al. 2016). Para diminuir a perda de biodiversidade e ainda suprir as necessidades humanas de produtos

1. Parte da Dissertação de Mestrado do primeiro Autor.

2. Universidade Estadual do Sudoeste da Bahia, Programa de Pós-Graduação em Ciências Ambientais, Centro de Ensino Pesquisa e Extensão Socioambiental, BR 415, km 03, s/n, 45700-000 Itapetinga, Brasil

3. Universidade Estadual do Sudoeste da Bahia, Departamento de Ciências Exatas e Naturais, BR 415, km 03, s/nº , 45700-000 Itapetinga, Bahia, Brasil

4. Universidade Estadual do Sudoeste da Bahia, Departamento de Ciências Naturais, Laboratório de Biodiversidade do Semiárido, Estrada do Bem Querer, km 04, s/n, 45083-900 Vitória da Conquista, Bahia, Brasil

5. Autor para correspondência: ruiasguimaraes@yahoo.com.br 
agrícolas, a importância de sistemas produtivos menos impactantes, que preservam parcialmente a biodiversidade destas florestas, vem sendo reconhecida internacionalmente por estudos recentes (Harvey et al. 2008, Gardner et al. 2009, Piasentin \& Gois 2016, Rolim et al. 2016). Tais estudos reconhecem que a conservação não pode depender exclusivamente da preservação de ecossistemas sem qualquer forma de exploração.

O cultivo de cacau no Estado da Bahia, região nordeste do Brasil, é um exemplo de cultura que possui reduzido impacto ambiental em sua produção que teve início na década de 1830 (Mendes-Costa 2012). A maior parte $(70 \%)$ desta cultura na região utiliza o modelo de cultivo conhecido como "cacaucabruca" (Franco et al. 1994). Esse sistema consiste na plantação do cacau sob a sombra de árvores do dossel da floresta original no espaço ocupado anteriormente pelo sub-bosque que é quase completamente retirado (Tavares 1979, Lobão \& Bispo 2004, Lobão et al. 2004, Lobão 2007). Os primeiros cacauicultores encontraram na cobertura da floresta a proteção necessária para diminuir a incidência de ventos e a perda de umidade do solo pela evaporação nas lavouras de cacau. Adicionalmente, como o cacaueiro é uma planta umbrófila, a manutenção parcial do dossel permite a proteção necessária para seu estabelecimento na sua fase inicial (Coutinho 1970) e para a formação e desenvolvimento dos frutos, proporcionando um sombreamento entre 30 a $60 \%$ de radiação direta (Batista \& Alvim 1981). Esta adaptação do cultivo às condições edafo-climáticas do sul da Bahia, conferiram a esta região o título de maior produtora de cacau do país, gerando riquezas, criando uma cultura própria, com consequente surgimento e crescimento de suas cidades (Goulart et al. 2014).

O sistema "cacau-cabruca" é considerado como modelo de exploração do solo que, diferentemente de outras silviculturas, dispensa a derrubada total da mata e pode representar uma opção de exploração sustentável dos ecossistemas tropicais (Nair 1993, Piasentin \& Góis 2016). Dessa forma, este modelo de cultivo do cacaueiro tem contribuído para a conservação de grande parte da biodiversidade da Mata Atlântica (Rice \& Greenberg 2000, Sambuichi 2002, Cassano et al. 2009, Piasentin \& Gois 2016, Rolim et al. 2016), que nessa região apresenta os mais elevados índices de biodiversidade do país, com até 456 espécies arbóreas registradas em um hectare (Araujo et al. 1998). Neste sentido, considerando que a composição e diversidade de espécies arbóreas em florestas tropicais variam em função de diversos fatores biológicos como área e tempo de regeneração (Santos et al. 2008, Rolim et al. 2016), e o importante papel das cabrucas para a conservação de um subconjunto de espécies da Mata Atlântica (Cassano et al. 2009, Piasentin \& Gois 2016, Rolim et al. 2016), o objetivo deste trabalho foi conhecer e avaliar a diversidade arbórea de cabrucas de Fazendas produtoras de cacau no centro-sul da Bahia e verificar se fatores como as áreas das propriedades e das cabrucas influenciam as comunidades arbóreas remanescentes.

\section{Material e métodos}

Área de estudo - O estudo foi realizado em áreas plantadas com cacaueiros no sistema cabruca localizadas em 10 fazendas dos municípios de Caatiba, Itororó e Firmino Alves, no Estado da Bahia (tabela 1). As áreas apresentam altitudes entre 285 e $590 \mathrm{~m}$. A temperatura média anual da região é de $23,5^{\circ} \mathrm{C}$, com clima tropical quente e úmido, tipo Aw de acordo com Köppen e Geiger, e com umidade relativa média de $89-90 \%$ e pluviosidade média anual de $865 \mathrm{~mm}$. As estações secas e chuvosas são nos meses de abril a outubro e novembro a março, respectivamente.

Coleta de dados - Em cada fazenda foram estabelecidas aleatoriamente nas áreas de cabruca três parcelas de $20 \mathrm{~m} \times 50 \mathrm{~m}$. Todos os indivíduos arbóreos presentes nas parcelas com diâmetro à altura do peito (DAP) acima de 9,5 cm, exceto os cacaueiros (Theobroma cacao L.) foram contados e identificados, bem como tiveram sua altura estimada visualmente. Material vegetativo e reprodutivo (flores e frutos) dos indivíduos arbóreos foi coletado e identificado por especialistas do Herbário Centro de Pesquisas do Cacau (CEPEC), utilizando-se bibliografia taxonômica específica (e.g. Lewis 1987, Souza \& Lorenzi 2008) e por comparação em herbário. As espécies foram discriminadas entre nativas e exóticas, de acordo com Flora do Brasil (2016) e com base no estudo de Sambuichi (2002).

Análise de dados - A diversidade de espécies para cada propriedade foi determinada pelo índice de Shannon-Wiener (H') (Krebs 1999). O quociente de mistura de Jentsch (QM), que representa o número total de espécies em relação ao número de indivíduos amostrados, foi calculado para cada uma das propriedades pela fórmula: $\mathrm{QM}=\mathrm{S} / \mathrm{n}$, onde $\mathrm{S}=$ número total de espécies e $\mathrm{n}=$ número de indivíduos (Brower \& Zar 1984).

A rarefação foi calculada utilizando a função de Hurlbert (1971). Para isso foi utilizado o valor da 
Tabela 1. Lista de espécies e número de indivíduos arbóreos inventariados em sistema cacau-cabruca em 10 fazendas localizadas nos municípios de Caatiba, Itororó e Firmino Alves no Estado da Bahia, Brasil. *Espécies exóticas. **Fazendas: 1. Ankilostomina, 2. Baixa Alegre, 3. Belo Horizonte I, 4. Belo Horizonte II, 5. Futurosa, 6. Maravilha, 7. Santo Antônio, 8. São Francisco, 9. São João e 10. Serra Dourada.

Table 1. Tree species and individuals found in cocoa-cabruca system in 10 cocoa farms in the municipality of Caatiba, Itororó and Firmino Alves, Bahia State, Brazil. *Exotic species. ** Farms: 1. Ankilostomina, 2. Baixa Alegre, 3. Belo Horizonte I, 4. Belo Horizonte II, 5. Futurosa, 6. Maravilha, 7. Santo Antônio, 8. São Francisco, 9. São João e 10. Serra Dourada.

\begin{tabular}{|c|c|c|c|c|c|c|c|c|c|c|}
\hline \multirow{2}{*}{ Família/Espécie } & \multicolumn{10}{|c|}{ Fazendas** } \\
\hline & 1 & 2 & 3 & 4 & 5 & 6 & 7 & 8 & 9 & 10 \\
\hline Anacardiaceae & & 1 & & & & 3 & & & 1 & \\
\hline Apterocarpus gardneri (Engl.) Rizinni & & & & & & & & & & \\
\hline Astronium macrocalix Engl. & & & & & & & 1 & & & \\
\hline Mangifera indica L.* & 1 & & & & 1 & 2 & & 1 & & \\
\hline Spondias dulcis G. Forst. & & 3 & & & & & & & & \\
\hline Spondias mombin L. & 1 & & & 1 & & & 1 & 3 & 7 & \\
\hline Annonaceae & & & & 1 & & & & & & \\
\hline Annona dolabripetala Raddi & & & & & & & & & & \\
\hline Annona mucosa (Jacq.) Baill. & & & & 1 & & & & & & \\
\hline $\begin{array}{l}\text { Arecaceae } \\
\text { Attalea oleifera } \text { Barb. Rodr. }\end{array}$ & & & & & & & 1 & & & \\
\hline Asteraceae & & & & & & & & & & 2 \\
\hline Vernonia sp. & & & & & & & & & & \\
\hline Bignoniaceae & & & & & & & 1 & & & \\
\hline $\begin{array}{l}\text { Handroanthus chrysotrichus (Mart. ex DC) } \\
\text { Mattos }\end{array}$ & & & & & & & & & & \\
\hline $\begin{array}{l}\text { Handroanthus impetiginosus (Mart. ex DC) } \\
\text { Mattos }\end{array}$ & & & & & & & & 1 & & \\
\hline $\begin{array}{l}\text { Boraginaceae } \\
\text { Cordia trichotoma (Vell.) Arráb. ex Steud. }\end{array}$ & 1 & & 2 & & & & & & & \\
\hline $\begin{array}{l}\text { Cannabaceae } \\
\quad \text { Trema micranta }(\text { L.) Blume }\end{array}$ & & & & 1 & & & & & & \\
\hline Combretaceae & & & & & & & & & & 2 \\
\hline $\begin{array}{l}\text { Terminalia brasiliensis (Cambess. ex A. St. } \\
\text {-Hil.) Eichler }\end{array}$ & & & & & & & & & & \\
\hline Euphorbiaceae & 2 & & & & & & & & 2 & \\
\hline Alchornea glandulosa Poepp. \& Endl. & & & & & & & & & & \\
\hline $\begin{array}{l}\text { Hevea brasiliensis (Willd. ex A. Juss.) Müll. } \\
\text { Arg.* }\end{array}$ & & & & & 1 & & & & & \\
\hline Mabea piriri Aubl. & & & & & 1 & & & & & \\
\hline Fabaceae & & & 1 & & & & & & 2 & 1 \\
\hline Albizia polycephala Benth. & & & & & & & & & & \\
\hline Bauhinia ovata (Bong) Vogel & & & & & & & & & & 1 \\
\hline Caesalpinia ferrea Mart. & & & & & & & 1 & & & \\
\hline Cassia grandis L.F. & & & & & & & & & & 1 \\
\hline Centrolobium robustum (Vell) Mart. ex Benth. & & & & & & & & & & 9 \\
\hline Centrolobium tomentosum Guillem. ex Benth. & & & & & & & 1 & 1 & & 8 \\
\hline Dalbergia nigra (Vell.) Allemão ex Benth. & & & & 1 & & & & & & 3 \\
\hline Enterolobium sp.(Vell.) Hauman & & 2 & & & 1 & & & 1 & & \\
\hline
\end{tabular}


Tabela 1 (continuação)

\begin{tabular}{|c|c|c|c|c|c|c|c|c|c|c|}
\hline \multirow{2}{*}{ Família/Espécie } & \multicolumn{10}{|c|}{ Fazendas** } \\
\hline & 1 & 2 & 3 & 4 & 5 & 6 & 7 & 8 & 9 & 10 \\
\hline Erythrina poeppigiana (Walp.) O.F. Cook & 1 & & 1 & 2 & 3 & & & 5 & 1 & 1 \\
\hline Lonchocarpus glabrescens Benth. & & & & & & & & & & 3 \\
\hline Plathymenia foliolosa Benth. & 1 & & 2 & & & 2 & & & 3 & \\
\hline Plathymenia reticulata Benth. & 3 & & & & & & & & & \\
\hline Platycyamus regnellii Benth. & & & 2 & & & & & & 2 & 8 \\
\hline Pterocarpus rohrii Vahl & & 1 & & & & & & & & \\
\hline Pterogyne nitens Tull & & & & & & & & & 2 & \\
\hline Schizolobium parahyba (Vell.) S.F. Blake & & & 1 & & 2 & & 15 & & 5 & \\
\hline Senna multijuga subsp. Lindlleyana (Gardner) & & & & & & 1 & & & 3 & 10 \\
\hline Swartzia macrostachya Benth. & & & & & & & & & 1 & \\
\hline Vataireopsis araroba (Aguiar) Ducke & 2 & & & & & & & & & \\
\hline Lauraceae & & & 1 & 5 & 2 & & & & & \\
\hline Licaria bahiana Kurz. & & & & & & & & & & \\
\hline Nectandra membranacea $(\mathrm{Sw})$ Griseb. & & & & 1 & & & & & & \\
\hline Persea americana Mill.* & & 1 & 2 & 1 & & 1 & & 2 & & \\
\hline Lecythidaceae & & & & & & & & 1 & & 1 \\
\hline Cariniana estrellensis (Raddi) Kuntze & & & & & & & & & & \\
\hline Cariniana legalis (Mart.) Kuntze & & & & & & 1 & & 2 & 1 & \\
\hline Lecythis pisonis (Mart.) Kuntze & & & & & & 1 & & 1 & & \\
\hline Malvaceae & & & & & & & & 1 & & 1 \\
\hline Cavanillesia umbellata Ruiz e Pav. & & & & & & & & & & \\
\hline $\begin{array}{l}\text { Eriotheca macrophylla (K. Schum.) A. } \\
\text { Robyns }\end{array}$ & & & 1 & & & & & & & \\
\hline Luehea divaricata Mart. & & & & & 2 & & & & & \\
\hline Meliaceae & 1 & & & & & & & & & \\
\hline Cedrela fissilis Vell. & & & & & & & & & & \\
\hline Moraceae & 4 & 2 & 13 & 2 & 10 & & & 10 & 7 & 11 \\
\hline Artocarpus heterophyllus Lam.* & & & & & & & & & & \\
\hline Ficus sp. & & & & & 1 & & 3 & & & 1 \\
\hline Rutaceae & & & & 1 & & & & & & \\
\hline Citrus sinensis L.* & & & & & & & & & & \\
\hline Zanthoxylum rhoifolium Lam. & & & & & 1 & & & & & \\
\hline Rubiaceae & & & & 1 & & & & & & \\
\hline Genipa americana L.* & & & & & & & & & & \\
\hline Phytolaccaceae & & 2 & & & 1 & & & & 1 & \\
\hline Gallesia integrilolia (Spreng.) Harms & & & & & & & & & & \\
\hline Solanaceae & & & & & & & & & & 8 \\
\hline Cestrum laevigatum Schltdl & & & & & & & & & & \\
\hline Urticaceae & & & & & & & & & 1 & \\
\hline Cecropia graziovii Snethl. & & & & & & & & & & \\
\hline Cecropia hololeuca Miq. & & & & & 1 & & & & & \\
\hline
\end{tabular}

menor abundância encontrada menos um indivíduo. Esta abordagem da rarefação gera a riqueza esperada de espécies para uma sub amostra aleatória de tamanho fixo.

A rarefação, densidade de indivíduos e diversidade foram relacionadas com a área das propriedades e área das cabrucas estudadas com o uso do teste de regressão linear múltipla. Para isso, os dados de área das propriedades e das cabrucas foram transformados em log para atender às premissas da regressão, que foram testadas com análise de resíduos. Os dados não 
apresentaram autocorrelação espacial segundo o teste I de Moran.

\section{Resultados}

Nas cabrucas estudadas foram encontrados 273 indivíduos arbóreos, pertencentes a 55 espécies de 20 famílias (tabela 1). A família mais representativa foi Fabaceae (19 espécies), que abrangeu 42,7 \% dos indivíduos e 34,2\% das espécies (tabela 1).

As espécies nativas representaram $89 \%$ das espécies e $73 \%$ dos indivíduos arbóreos das cabrucas (tabela 1). Contudo, a espécie exótica Artocarpus heterophyllus Lam. foi a que apresentou o maior número de indivíduos e frequência de ocorrência. Essa espécie representou $21,6 \%$ dos indivíduos arbóreos amostrados e ocorreu em oito fazendas. Schizolobium parahyba (Vell.) S.F. Blake foi a segunda espécie com mais indivíduos, representando $11,1 \%$ dos indivíduos e Erythrina poeppigiana (Walp.) O.F. Cook foi a segunda espécie mais frequente, ocorrendo em sete fazendas. Apenas uma espécie ameaçada de extinção foi encontrada, Dalbergia nigra (Vell.) Allemão ex Benth., registrada em duas fazendas, e cujo número de indivíduos representou apenas $1,4 \%$ do total amostrado.

Cada hectare de cabruca avaliado possui no mínimo 40 a até 236 indivíduos arbóreos $(91 \pm 59$ indivíduos; tabela 2) e 23 a 60 espécies $(37 \pm 13$ espécies; tabela 2). A diversidade de espécies variou de 1,3 a 2,5. O QM apresentou uma proporção de uma espécie arbórea para cada dois indivíduos amostrados.

As cabrucas ocupam de 13,1 a $100 \%(44,4 \pm$ $32,2 \%$ ) da área das fazendas. Apesar disso, o tamanho dessas propriedades e suas respectivas áreas de cabrucas não influenciaram significativamente a riqueza de espécies, densidade de indivíduos por hectare ou diversidade (tabela 3 ).

\section{Discussão}

A riqueza de espécies de árvores do sistema de cultivo cacau-cabruca na região estudada, no centrosul da Bahia, é similar em número de espécies ao registrado para outras áreas com o mesmo tipo de sistema agroflorestal mais ao sul do Estado (Sambuichi 2002, 2006). A família Fabaceae, mais representativa em número de indivíduos e espécies neste estudo, corrobora outros levantamentos que reportam esse táxon como um dos mais frequentes em florestas ombrófilas densas (Guilherme et al. 2004, Thomas et al. 2009). Contudo, a espécie mais comum em número de indivíduos e ocorrência nas cabrucas estudadas foi a espécie exótica A. heterophyllus, a jaqueira, da família Moraceae. Essa espécie é abundante e freqüente em remanescentes de Mata Atlântica (Hummel 1995), tanto que atualmente é a espécie mais usada pelo mico-leão-da-caradourada como alimento devido à sua disponibilidade ao longo do ano (Oliveira et al. 2011). A grande representatividade desta espécie nesta floresta e nas

Tabela 2. Características estruturais e de diversidade arbórea de cabrucas de 10 fazendas produtoras de cacau do centro-sul baiano. N/ha: número de indivíduos por hectare, S/ha: riqueza de espécies por hectare, H': índice de Shannon-Wiener e QM: quociente de mistura de Jenstch.

Table 2. Structural characteristics and tree diversity of 10 cocoa farms in the central Southern region of Bahia State. N/ha: individuals number per hectare, S/ha: species richness per hectare, H': Shannon-Wiener index and QM: Jentsch' mixture quotient.

\begin{tabular}{lccccccc}
\hline Fazenda & $\begin{array}{c}\text { Área total } \\
\text { (ha) }\end{array}$ & $\begin{array}{c}\text { Área de cabruca } \\
\text { (ha) }\end{array}$ & N/ha & S/ha & H' & QM & $\begin{array}{c}\text { Município no Estado da } \\
\text { Bahia }\end{array}$ \\
\hline Ankilostomina & 15 & 15 & 53 & 30 & 2,2 & 0,56 & Itororó \\
Baixa Alegre & 10 & 10 & 40 & 23 & 1,9 & 0,58 & Caatiba \\
Belo Horizonte I & 31 & 14,5 & 83 & 30 & 1,8 & 0,36 & Itororó \\
Belo Horizonte II & 106 & 66 & 60 & 40 & 2,3 & 0,67 & Itororó \\
Futurosa & 60 & 20 & 90 & 43 & 2,2 & 0,48 & Caatiba \\
Maravilha & 370,5 & 80 & 40 & 27 & 1,8 & 0,67 & Firmino Alves \\
Santo Antônio & 228,8 & 30 & 77 & 23 & 1,3 & 0,30 & Itororó \\
São Francisco & 85 & 15 & 97 & 40 & 2,1 & 0,41 & Firmino Alves \\
São João & 62 & 15 & 133 & 53 & 2,4 & 0,40 & Itororó \\
Serra Dourada & 80 & 20 & 237 & 60 & 2,5 & 0,25 & Itororó \\
\hline
\end{tabular}


Tabela 3. Regressão múltipla entre parâmetros de diversidade arbórea em cabrucas com a área total e área das cabrucas de 10 fazendas produtoras de cacau do centro-sul baiano, Brasil.

Table 3. Multiple regression among tree diversity parameters in cabrucas with the total area and the cabrucas areas of 10 cocoa farms of the central Southern region of Bahia State, Brazil.

\begin{tabular}{ccccc}
\hline Modelo & F & Graus de Liberdade & $p$ & $\mathrm{R}^{2}$ \\
\hline Rarefação & 1,557 & 2 e 7 & 0,276 & 0,308 \\
Densidade de indivíduos & 1,194 & 2 e 7 & 0,358 & 0,254 \\
Diversidade Shannon-Wiener & 0,154 & 2 e 7 & 0,860 & 0,042 \\
\hline
\end{tabular}

cabrucas ocorre devido à sua introdução intencional no passado, para consumo humano dos frutos, uso da madeira, para proporcionar sombra e pela fácil dispersão natural dos seus diásporos no ambiente (Hummel 1995, Sambuichi et al. 2012, Piasentin et al. 2014).

Outras duas espécies, S. parahyba e E. poeppigiana também foram destaque entre as espécies arbóreas registradas, sendo as mais abundantes e frequentes, respectivamente nas cabrucas. A espécie S. parahyba é de ocorrência comum em vegetação secundária e indicada para plantio em áreas para recuperação de matas ciliares (Ferreira et al. 2007). Já a espécie E. poeppigiana, foi, durante muito tempo, utilizada no sombreamento do cacaueiro, por sua precocidade e pela capacidade da espécie em proporcionar a incorporação de nitrogênio no solo (Van Belle et al. 2003) por meio da associação com bactérias diazotróficas e fungos micorrízicos, característica compartilhada por outras espécies do gênero (Silva et al. 2007). Essa espécie, porém, vem sendo substituída por outras espécies com maior valor econômico e alimentício (Marques et al. 2003). Existe uma tendência atual de substituição e redução da densidade da flora nativa das cabrucas por espécies exóticas para consumo ou venda (Sambuichi 2006, Sambuichi et al. 2012, Cassano et al. 2014, Piasentin et al. 2014). Além disso, alguns cacauicultores consideram que um sombreamento em excesso dos cacaueiros aumenta a infestação das plantações por doenças como a vassoura-de-bruxa (Moniliophthora perniciosa), diminuindo a produção, bem como promovem um maior crescimento e desenvolvimento longitudinal das plantas, e esse aumento em altura dificulta a colheita do cacau (Piasentin et al. 2014).

A densidade de árvores encontrada nas fazendas apresenta valores entre os mais altos registrados na literatura para cabrucas baianas (Sambuichi 2006). Lobão et al. (2004) consideram cabrucas de baixa densidade aquelas com densidade entre 25 e 50 ind.ha ${ }^{-1}$, de média densidade entre 50 e 85 ind $\mathrm{ha}^{-1}$ e de alta densidade acima de 85 ind.ha $^{-1}$. Neste sentido, $80 \%$ das fazendas estudadas apresentaram média e alta densidade arbórea nas cabrucas. Esse resultado é contrário a alguns dos métodos de implantação e manejo do cacau recomendados pela Comissão Executiva do Plano da Lavoura Cacaueira (CEPLAC), que sugere de 25-30 ind.ha ${ }^{-1}$ a densidade ideal de árvores de sombra em áreas de cabruca, como forma de aumentar a produtividade dos cacaueiros (Piasentin \& Saito 2014). O cultivo do cacau com pouco sombreamento ou a pleno sol realmente aumenta a produtividade desta cultura, contudo, demanda mais irrigação, nutrientes e controle de pragas, uma vez que a radiação solar direta aumenta o estresse hídrico das plantas (Arévalo et al. 2007, Piasentin et al. 2014).

Embora a diversidade de espécies arbóreas deste estudo seja inferior a observada em outras áreas de cabruca (Lobão 2007, Sambuichi 2002, Sambuichi 2006) e apenas uma espécie ameaçada de extinção tenha sido registrada, D. nigra, um elevado valor de QM foi encontrado, e conferindo às cabrucas da região alta heterogeneidade florística, de acordo com Finol (1975). Este autor propôs que em florestas naturais tropicais o QM seria em torno da proporção de uma espécie encontrada a cada nove indivíduos amostrados. De fato, uma vez que a região sul da Bahia é considerada como uma das regiões mais biodiversas do mundo, principalmente quanto à diversidade vegetal (Thomas et al. 2008), a elevada riqueza de espécies arbóreas registradas nas cabrucas era esperada, mesmo tratando-se de uma agrofloresta. Dessa forma, ainda que as cabrucas mantenham menos de $10 \%$ da quantidade de árvores de florestas naturais (Sambuichi 2002), essas áreas podem constituir importantes bancos genéticos para espécies arbóreas, são importantes como corredores ecológicos e refúgio para a fauna, protegem recursos hídricos e constituem-se em uma estratégia complementar para conservação de espécies da Mata Atlântica, diferentemente de outros sistemas produtivos, como a agricultura tradicional ou a silvicultura, que retiram 
toda a vegetação natural para o plantio de culturas (Rolim \& Chiarello 2004, Sambuichi 2002).

De maneira geral, a ausência de relação entre as características das fazendas com os parâmetros de diversidade avaliados aqui, bem como a alta densidade de indivíduos e QM registrados evidenciam um manejo diferenciado realizado pelos proprietários ao longo dos anos de cultivo do cacau, desde sua implantação. As variações no manejo das cabrucas ocorrem por diversos fatores, como pela retirada de árvores que morreram naturalmente (Rolim \& Chiarello 2004), para uso medicinal ou como lenha (Sambuichi et al. 2012, Piasentin et al. 2014), para venda devido ao valor econômico das árvores (Piasentin et al. 2014) ou pela introdução de espécies exóticas para consumo (Hummel 1995, Piasentin et al. 2014). Além disto, a grande representatividade de espécies nativas em número de espécies e indivíduos nas cabrucas refletem a opção dos agricultores em manter essas espécies nas cabrucas.

Segundo Piasentin et al. (2014), muitos cacauicultores não interferem na regeneração de espécies de madeira de lei durante o manejo dos cacaueiros, mas muitas vezes priorizam a conservação de algumas poucas espécies. Essa prática de manejo, em longo prazo, pode comprometer a manutenção da diversidade do dossel das cabrucas. Adicionalmente, os mesmos autores ressaltam que o principal fator para a seleção das espécies arbóreas a serem mantidas nas cabrucas ainda é a geração de renda, e que a exploração de espécies de madeira de lei tem aumentado nos últimos anos em decorrência da crise da cultura do cacau (Piasentin et al. 2014). Mesmo que a manutenção de determinadas espécies arbóreas possa ser feita como uma reserva para uso futuro da madeira pelos proprietários, ainda existem aqueles que mantêm as árvores nativas nas cabrucas, pois visualizam outros benefícios dessas espécies além do valor madeireiro. Geralmente são espécies mais longevas, que oferecem menor risco de queda sobre a plantação, proporcionam sombra mais esparsa por serem mais altas e mantêm fauna associada mais diversificada otimizando o controle biológico de pragas (Sambuichi 2002, Rolim \& Chiarello 2004, Cassano et al. 2009, Sambuichi et al. 2012, Piasentin et al. 2014).

Aqui demonstramos que o sistema de cultivo cacau-cabruca das fazendas da região centro-sul da Bahia apresenta alta heterogeneidade, densidade e riqueza de espécies arbóreas em comparação com outros estudos em outras regiões com o mesmo tipo de agrofloresta (Sambuichi 2002). Porém, esses parâmetros de diversidade arbórea não são influenciados pela área total das propriedades ou das cabrucas, uma vez que cada fazenda produtora de cacau tem sido manejada de maneira diferente pelos proprietários. Como a estimativa de que as áreas de cobertura original da Mata Atlântica no sul da Bahia é de apenas 4\% (Thomas et al. 2008), nossos resultados corroboram estudos anteriores que ressaltam que o sistema cacau-cabruca é um uso da terra mais sustentável para a conservação pelo menos parcial da biodiversidadeem propriedades rurais do que outras atividades agrícolas (Cassano et al. 2009, Schroth et al. 2011, Piasentin \& Gois 2016). Essas agroflorestas podem manter grande número de espécies da flora e fauna nativas da região (Cassano et al. 2014), e talvez sejam os únicos habitats disponíveis para a sobrevivência de espécies arbóreas nativas endêmicas e ameaçadas de extinção, e que nos remanescentes de floresta já foram praticamente extintas (Sambuichi 2002, Sambuichi et al. 2008), como é o caso da espécie de madeira de lei $D$. nigra, encontrada em duas fazendas (Guedes et al. 2011). Além disso, sistemas de manejo das cabrucas que otimizem seu papel de conservação da biodiversidade são desejáveis e este relevante papel desses ecossistemas manejados deve ser melhor divulgado para que possa ser reconhecido.

\section{Agradecimentos}

Os autores agradecem ao herbário André Mauricio Vieira de Carvalho do Centro de Pesquisa do Cacau na Comissão Executiva do Planejamento da Região Cacaueira (CEPLAC), pelo auxílio na identificação do material botânico. Agradecimentos especiais aos proprietários das fazendas estudadas: José Valmir, Addya Galvão, Elandir Bezerra, José Balbino, Jaime Valverde, Eduardo Paixão, Luciana Pedreira, José Pereira de Jesus, Alaide dos Santos e Adelmo Cardoso pela permissão na realização do trabalho e auxilio nas coletas de dados, e a Raymundo José de Sá Neto pela contribuição na análise dos dados e em todo o manuscrito.

\section{Literatura citada}

Araujo, M., Alger, K., Rocha, R. \& Mesquita, C. 1998. A Mata Atlântica do Sul da Bahia: Situação atual, ações e perspectivas. Caderno 08. Conselho Nacional da Reserva da Biosfera da Mata Atlântica, São Paulo.

Arévalo, E., Ram, A., Monteiro, W.R.\& Valle, R.R. 2007. Integração de práticas de manejo no cultivo de cacau. In: R.R. Valle (ed.). Ciência, Tecnologia e Manejo do Cacaueiro. CEPLAC/CEPEC, Ilhéus. 
Batista, L.P. \& Alvim, R. 1981. Efeitos da intensidade luminosa e do fenótipo sobre o crescimento emaltura do fuste do cacaueiro. Revista Theobroma 11: 61-76.

Brower, J.E. \& Zar, J.H. 1984. Field and laboratory methods for general ecology. 2 ed. C. Brown Publishers, Dubuque.

Cassano, C.R., Schroth, G., Faria, D. Delabie, J.H.C. \& Bede, L. 2009. Landscape and farm scale management to enhance biodiversity conservation in the cocoa producing region of southern Bahia, Brazil. Biodiversity and Conservation 18: 577-603.

Cassano, C.R., Schroth, G., Faria, D., Delabie, J.H.C., Bede, L., Oliveira, L.C. \& Mariano-Neto, E. 2014. Desafios e recomendações para a conservação da biodiversidade na região cacaueira do sul da Bahia. Boletim técnico ${ }^{\circ}$ 205. CEPLAC/CEPEC, Ilhéus.

Coutinho, A. 1970. A importância do sombreamento na cultura do cacaueiro. In: Curso Internacional de Cacau, Itabuna, BA. Comissão Executiva do Plano de Recuperação Econômico Rural da Lavoura Cacaueira, Itabuna, BA (Brasil), Itabuna.

Ferreira, R.A., Oliveira, L.M., Tonetti, O.A.O. \& Davide, A.C. 2007. Comparação da viabilidade de sementes de Schizolobium parahyba (Vell.) Blake - Leguminosae, Caesalpinoideae, pelos testes de germinação e tetrazólio. Revista Brasileira de Sementes 29: 73-79.

Finol, U.H. 1975. La silvicultura em la Orinoquia Venezolana. Revista Forestal Venezolana 18: 37-114.

Flora do Brasil. 2016. Jardim Botânico do Rio de Janeiro. Disponível em http://floradobrasil.jbrj.gov.br (acesso em 28-V-2016).

Franco, M., Holz, B., Kaule, G., Kleyer, M., Menezes, M., Pereira, J. \& Trevisan, S. 1994. Program of the environmental development of the rainforest region in Bahia, Brazil - development of a methodology. InstitutfürLandschaftsplanung und Ökologie, University Stuttgart, Stuttgart.

Gardner, T.A., Barlow, J., Chazdon, R., Ewers, R.M., Harvey, C.A., Peres, C.A. \& Sodhi, N.S. 2009. Prospects for tropical forest biodiversity in a humanmodified world. Ecology Letters 12: 561-582.

Goulart, L.A., Segundo, G.S.A., Junior, M.F.S., Uetanabaro, A.P.T. 2014. Cacau da região sul da Bahia e a perspectiva histórica de uma indicação geográfica. Cadernos de Prospecção 7: 632-639.

Guedes, R.S., Alves, E.U., Gonçalves, E.P., França, P.R.C., Moura, M.F. \& Santos, S.S. 2011. Germinação de sementes de Dalbergia nigra (Vell.) Fr. All. Maringá 33: 445-450.

Guilherme, F.A.G., Morelato, L.P.C. \& Assis, M.A. 2004. Horizontal and vertical tree community structure in a lowland Atlantic Rain Forest, Southeastern Brazil. Revista Brasileira de Botânica 27: 725-737.
Harvey, C.A., Komar, O., Chazdon, R., Ferguson, B.G., Finegan, B., Griffith, D.M., Martínez-Ramoz, M., Morales, H., Nigh, R., Soto-Pinto, L., Van Breugel, M. \& Wishnie, M. 2008. Integrating Agricultural Landscapes with Biodiversity Conservation in the Mesoamerican Hotspot. Conservation Biology 22: 8-15.

Hummel, M. 1995. Botanical analysis of the shade tree population in two cabruca cocoa plantations in southern Bahia, Brazil. 1995. Tese de Doutorado, University of Stuttgart, Germany.

Hurlbert S.H. 1971. The nonconcept of species diversity: a critique and alternative parameters. Ecology 52:577-86.

Krebs, C.J. 1999. Ecological Methodology. Benjamin/ Cummings, California.

Lewis, G.P. 1987. Legumes of Bahia. Royal Botanic Gardens, Kew.

Lobão, D.E. \& Bispo, K.C. 2004. Uso múltiplo da propriedade rural-Gestão, Conservação Produtiva e Uso. Disponível em http:// www.ceplac.gov.br/radar/Artigos/ artigo36.htm (acesso em 13-X-2015).

Lobão, D.E. 2007. Agrossistema Cacaueiro da Bahia: Cacau cabruca e fragmentos florestais na conservação de espécies arbóreas. Tese de Doutorado. Faculdade de Ciências agrárias e Veterinárias, Unesp, cidade.

Lobão, D.E., Setenta, W.C. \& Valle, R.R. 2004. Sistema agrossilvicultural cacaueiro- modelo de agricultura sustentável. Agrossilvicultura 1: 163-173.

Marques, J.R.B. 2003. Substituição da Eritrina por outras espécies arbóreas de valor econômico - Um enfoque sustentável de Modernização Agrícola. In: Semana do Fazendeiro, Uruçuca.

Mendes-Costa, F. 2012. Políticas públicas e atores sociais na evolução da cacauicultura baiana. Opção Editora, Vila Velha.

Nair, P.K.R. 1993. An Introducion do Agroforestry. Kluwer academic Publishers, Gainesville.

Oliveira, L.C., Neves, L.G., Raboy, B.E. \& Dietz, J.M. 2011. Abundance of Jackfruit (Artocarpus heterophyllus) Affects Group Characteristics and Use of Space by Golden-Headed Lion Tamarins (Leontopithecus chrysomelas) in Cabruca Agroforest. Environmental Management: 48: 248-262.

Oliveira-Filho, A.T. \& Fontes, M.A.L. 2000. Patterns of Floristic Differentiation among Atlantic Forests in Southeastern Brazil and the Influence of Climate. Biotropica 32: 793-810.

Piasentin, F.B. \& Góis, S.L.L. 2016. Conservação de remanescentes florestais no Brasil: considerações sobre os principais instrumentos de gestão ambiental. Desenvolvimento e Meio Ambiente 36: 115-134.

Piasentin, F.B. \& Saito, C.H. 2014. Os diferentes métodos de cultivo de cacau no sudeste da Bahia, Brasil: aspectos históricos e percepções. Boletim do Museu Paraense Emílio Goeldi. Ciências Humanas 9: 61-78. 
Piasentin, F.B., Saito, C.H. \& Sambuichi, R.H.R. 2014. Preferências locais quanto às árvores do sistema cacaucabruca no sudeste da Bahia. Ambiente \& Sociedade 17: $55-78$.

Rice, R.A. \& Greenberg, R. 2000. Cacao Cultivation and the Conservation of Biological Diversity. Ambio 29: 167- 173 .

Rolim, S.G. \&Chiarello, A.G. 2004. Slow death of Atlantic forest trees in cocoa agroforestry in south-eastern Brazil. Biodiversity and Conservation 13: 2679-2694.

Rolim, S.G., Sambuichi, R.H.R., Schroth, G., Nascimento, M.T. \& Gomes, J.M.L. 2016. Recovery of forest and phylogenetic structure in abandoned cocoa agroforestry in the Atlantic forest of Brazil. Environmental Management.

Sambuichi, R.H.R. 2002. Fitossociologia e diversidade de espécies arbóreas em cabruca (Mata Atlântica raleada sobre plantação de cacau) na região sul da Bahia, Brasil. Acta Botânica Brasilica 16: 89-101.

Sambuichi, R.H.R. 2006. Estrutura e dinâmica do componente arbóreo em área de cabruca na região cacaueira do sul da Bahia, Brasil. Acta Botânica Brasilica 20: 943-954.

Sambuichi, R.H.R., Oliveira, R.M., Mariano-Neto E., Thévenin, J.M.R., Jesus-Junior, C.P., Oliveira, R.L. \& Pelição, M.C. 2008. Conservation status of ten endemic trees from the Atlantic forest in the south of Bahia-Brazil. Natureza e Conservação 6: 208-225.

Sambuichi, R.H.R., Vidal, D.B., Piasentin, F.B., Jardim, J.G., Viana, T.G., Menezes, A.A., Mello, D.L.N., Ahnert, D. \& Baligar, V.C. 2012. Cabruca agroforests in southern Bahia, Brazil: tree component, management practices and tree species conservation. Biodiversity and Conservation 21: 1055-1077.
Santos, B.A., Peres, C.A., Oliveira, M.A., Grillo, A., Alves-Costa, C.P. \& Tabarelli, M. 2008. Drastic erosion in functional attributes of tree assemblages in Atlantic forest fragments of northeastern Brazil. Biological conservation 141: 249-260.

Schroth, G., Faria, D., Araujo, M., Bede, L., Van Bael, S.A., Cassano, C.R., Oliveira, L.C. \& Delabie, J.H.C. 2011.Conservation in tropical landscape mosaics: the case of the cacao landscape of southern Bahia, Brazil. Biodiversity and Conservation 20: 1635-1654.

Silva, G.T.A., Resende, A.S., Campello, E.F.C., Dias, P.F. \& Franco, A.A. 2007. O papel da fixação biológica de nitrogênio na sustentabilidade de sistemas agroflorestais. Embrapa Agrobiologia, Seropédica, cidade.

Souza, V.C. \& Lorenzi, H. 2008. Botânica Sistemática - Guia ilustrado para a identificação das famílias de Fanerógamas nativas e exóticas no Brasil, baseado em APG II. Plantarum, Nova Odessa.

Tavares, L.H.D. 1979. História da Bahia. 6 ed. Ática, São Paulo.

Thomas, W.W., Carvalho, A.M.V., Amorim, A.M., Hanks, J.G. \& Santos, T.S. 2008. Diversity of woody plants in the Atlantic forest of southern Bahia, Brazil. In: W.W. Thomas (ed.).The Atlantic Coastal Forest of Northeastern Brazil. The New York Botanical Garden Press, New York.

Thomas, W.W., Jardim, J.G., Fiaschi, P., MarianoNeto, E. \& Amorim, A.M.A. 2009. Composição florística e estrutura do componente arbóreo de uma área transicional de Floresta Atlântica no Sul da Bahia, Brasil. Revista Brasileira de Botânica 32: 41-54.

Van Belle, J.F., Lobão, D.E. \& Herrera, S. 2003. La forêtdense humilde atlantiqueduBrésil et lesystèmecacao-cabruca bahianais. Parcs e Réserves 58: 22-28. 